\title{
BMJ Open Examining different measures of multimorbidity, using a large prospective cross-sectional study in Australian general practice
}

\author{
Christopher Harrison, Helena Britt, Graeme Miller, Joan Henderson
}

To cite: Harrison $\mathrm{C}$, Britt $\mathrm{H}$, Miller G, et al. Examining different measures of multimorbidity, using a large prospective crosssectional study in Australian general practice. BMJ Open 2014;4:e004694.

doi:10.1136/bmjopen-2013004694

- Prepublication history for this paper is available online. To view these files please visit the journal online (http://dx.doi.org/10.1136/ bmjopen-2013-004694)

Received 16 December 2013 Revised 30 May 2014 Accepted 17 June 2014

CrossMark

Family Medicine Research Centre, School of Public Health, University of Sydney, Sydney, Australia

Correspondence to Christopher Harrison; christopher.harrison@sydney. edu.au

\section{ABSTRACT}

Objectives: Prevalence estimates of multimorbidity vary widely due to inconsistent definitions and measurement methods. This study examines the independent effects on prevalence estimates of how 'disease entity' is defined - as a single chronic condition or chapters/domains in the International Classification of Primary Care (V.2; ICPC-2), International Classification of Disease (10th revision; ICD-10) or the Cumulative IIIness Rating Scale (CIRS), the number of disease entities required for multimorbidity, and the number of chronic conditions studied.

Design: National prospective cross-sectional study. Setting: Australian general practice.

Participants: 8707 random consenting deidentified patient encounters with 290 randomly selected general practitioners.

Main outcome measures: Prevalence estimates of multimorbidity using different definitions.

Results: Data classified to ICPC-2 chapters, ICD-10 chapters or CIRS domains produce similar multimorbidity prevalence estimates. When multimorbidity was defined as two or more (2+) disease entities: counting individual chronic conditions and groups of chronic conditions produced similar estimates; the 12 most prevalent chronic conditions identified about $80 \%$ of those identified using all chronic conditions. When multimorbidity was defined as $3+$ disease entities: counting individual chronic conditions produced significantly higher estimates than counting groups of chronic conditions; the 12 most prevalent chronic conditions identified only two-thirds of patients identified using all chronic conditions.

Conclusions: Multimorbidity defined as 2+ disease entities can be measured using different definitions of disease entity with as few as 12 prevalent chronic conditions, but lacks specificity to be useful, especially in older people. Multimorbidity, defined as $3+$, requires more measurement conformity and inclusion of all chronic conditions, but provides greater specificity than the $2+$ definition. The proposed concept of "complex multimorbidity", the co-occurrence of three or more chronic conditions affecting three or more different body systems within one person without defining an index chronic condition, may be useful in identifying high-need individuals.
Strengths and limitations of the study

- A large, representative, prospective study of multimorbidity, involving 290 general practitioners and 8707 patients, allowed testing of the independent effect of variables on prevalence estimates, something not possible with systematic reviews.

- This study investigated all chronic conditions, not a selection of conditions.

- This study used the general practitioner as an 'expert interviewer', drawing on the patient's knowledge, the patient's health record and their own knowledge to indicate the patient's current chronic conditions. Most multimorbidity studies rely on only one of these sources of data.

- This study only considered chronic conditions, whereas some authors now include acute conditions when defining multimorbidity.

\section{INTRODUCTION}

Research into the coexistence of multiple chronic health conditions in an individual was initially concerned with comorbidity, defined as "the existence or occurrence of any distinct additional disease entity in a patient who has the index disease under study." However, since the early 1990s, interest has progressed to 'multimorbidity', commonly defined as the "co-occurrence of two or more diseases within one person without defining an index disease." $"$

Interest in multimorbidity is growing due to its expected increase resulting from the ageing of the world's population. ${ }^{3} 4$ Studies have shown that multimorbidity is associated with increased patient mortality, demand on health resources, complexity of care and reduced patient quality of life. ${ }^{5}{ }^{6}$ However, prevalence estimates of multimorbidity have ranged from $3.5 \%^{7}$ to $98.5 \%,{ }^{8}$ the wide variance thought to be due to the lack of standards defining multimorbidity and how it is measured. A recent systematic review found 
132 definitions involving 1631 different criteria. ${ }^{9}$ There have been many calls for standards and guidelines for research into multimorbidity. ${ }^{10-12}$ Recent systematic reviews have raised specific issues regarding the way multimorbidity is defined and/or measured. ${ }^{11}{ }^{12}$

The first issue is the number of conditions studied. Fortin $e t a l^{11}$ found that this ranged from five to all conditions. Diederichs $e t a l^{12}$ reported a range of 4-102 conditions (mean 18.5 and median 14) and suggested that conditions may be chosen for pragmatic reasons (such as data availability), as the majority of authors did not give reasons for their selection. Where they did, the most common was those conditions with a high prevalence or high impact on patients. ${ }^{12}$ Diederichs $e t$ al ${ }^{12}$ and Fortin et $a l^{11}$ suggested that studies considering only a few conditions produced lower prevalence estimates than those examining many conditions. Diederichs $e t a l^{12}$ suggested a list of 11 chronic conditions prevalent in the elderly as a minimum (cancer, diabetes mellitus, depression, hypertension, myocardial infarction, chronic ischaemic heart disease, heart arrhythmias, heart insufficiency, stroke, chronic obstructive pulmonary disease and arthritis). Fortin et $a l^{11}$ suggested that any 12 prevalent conditions should suffice to measure multimorbidity accurately.

The second issue is how 'disease entity' was defined in multimorbidity studies. Ideally, morbidities being counted should be 'distinct' disease entities. However, disease entities used across studies varied from very specific conditions to groups of conditions. Even Diederichs et $a l \mathrm{~s}^{12}$ suggested list (above) includes some disease entities that are groups of conditions (such as arthritis and cancer) and some very specific, closely related conditions (eg, myocardial infarction and chronic ischaemic heart disease). It is debatable whether myocardial infarction and chronic ischaemic heart disease should be considered as two separate disease entities in measuring multimorbidity. Some multimorbidity studies have tried to overcome this problem by only counting chronic conditions that affect different body systems, to ensure that the count was of distinct disease entities. ${ }^{4}{ }^{13}$ These studies used the Cumulative Illness Rating Scale (CIRS) ${ }^{14}$ domains to group chronic conditions by body system. ${ }^{4} 13$ Fortin $e t a l^{11}$ suggested that while the use of the CIRS needed further research, this approach may simplify coding and data collection. The impact of counting the different body systems affected by chronic conditions on multimorbidity prevalence estimates is not known.

Most primary care-based multimorbidity studies rely on a health record review. ${ }^{11}$ A disadvantage of using CIRS in such reviews is that it requires additional mapping of diagnoses from the classification system in which the health records were coded. The two most commonly used disease classification systems are the International Classification of Primary Care (V.2; ICPC-2) ${ }^{15}$ and the International Classification of Disease (10th revision; ICD-10). ${ }^{16}$ ICPC-2 is used in primary care, and its chapters (with the exception of 'General and unspecified' and 'Social' chapters) are body system- based, following the principle that localisation takes precedence over aetiology. ${ }^{16}$ ICD-10 is primarily used in hospitals and its chapters axes include body systems, aetiology and 'others'. ${ }^{16}$ ICD-10 lacks specificity for classification of undiagnosed problems or symptoms, both of which are commonly managed in primary care. ${ }^{17}$ This has meant that data from primary healthcare records classified in the two systems have looked very different in the past. However, since most multimorbidity studies examine only chronic conditions, this problem may be avoided when conditions are grouped at the chapter level. It is not known whether counting disease entities from different CIRS domains, ICPC-2 or ICD-10 chapters produces comparable multimorbidity prevalence estimates.

The third issue is the number of disease entities required to define multimorbidity. Originally, multimorbidity was defined as two or more (2+) disease entities, but recently there has been debate about whether three or more (3+) may be a better measure. Fortin et $a l^{11}$ argue that using 2+ disease entities identifies such a high proportion of patients as multimorbid that the measure lacks specificity. They found that age-specific prevalence of multimorbidity using the $2+$ definition produced an ' $\mathrm{S}$ ' shaped curve with a flat plateau for older ages. When using $3+$, the increase in prevalence by age was more linear, with greater differentiation in older age groups. The authors further argued that using $3+$ disease entities results in a lower prevalence estimate, is likely to identify patients with greater health needs and is therefore more useful to clinicians. ${ }^{11}$ They recommended further research to test the 3+ definition of multimorbidity. ${ }^{11}$

The current study was conducted in Australian general practice. Australia's universal medical insurance scheme, Medicare, fully or partially covers the individual's cost of visits to general practitioners (GPs). GPs provide the bulk of primary medical care and act as gatekeepers to government-subsidised healthcare from other medical specialists. There are no patient lists and patients are free to visit multiple GPs and practices as they choose.

Our study examines how multimorbidity prevalence estimates are affected by: the number of chronic conditions studied; how a disease entity is defined; and the minimum number of disease entities required to define multimorbidity. We use a large Australian general practice-based prospective multimorbidity study, which allows us to examine the effect of each of these variables on multimorbidity prevalence estimates while controlling for other confounding variables, an approach not possible in systematic reviews.

\section{METHOD}

The BEACH (Bettering the Evaluation And Care of Health) programme is a continuous, national crosssectional survey of general practice activity in Australia. ${ }^{17}$ 
Each year, an ever-changing sample of about 1000 GPs is randomly selected, and each GP records information about encounters with 100 consecutive consenting patients on structured paper forms. ${ }^{17}$

In substudies of BEACH, the GP records information additional to the encounter data, in discussion with the patient. The full methods for this substudy are reported elsewhere. ${ }^{18}$ In brief, it measured the prevalence of diagnosed chronic conditions in patients attending general practice in Australia. Over three 5-week recording periods (August 2008-May 2009), 375 sampled GPs were asked to record all diagnosed chronic conditions for each of 30 consecutive patients on 30 bespoke forms within their 100 BEACH records. A sample of the instruction sheet and recording form can be found at www.http://sydney. edu.au/medicine/fmrc/publications/sand-abstracts/132Multimorbidity.pdf

GPs were asked, "Does the patient have any of the following chronic diseases/problems?" Common chronic conditions were listed (tick boxes) with additional free text fields to record other unlisted chronic conditions. A 'no chronic conditions' option was also provided. Listed chronic conditions were primarily those most frequently managed in Australian general practice ${ }^{17}$ and were inclusions in O'Halloran et $a l \mathrm{~s}^{19}$ definition of chronic conditions. The free text options relied on GPs' judgement of whether a condition was chronic in this patient. GPs were instructed to "Use your own knowledge, patient knowledge and health records as you see fit, in order to answer these questions." Additional free text chronic conditions were coded using the ICPC-2 PLUS terminology ${ }^{20}$ which automatically classified them into ICPC-2. ${ }^{15}$ All chronic conditions were classified to ICD-10 chapters $^{16}(\mathrm{n}=20)$, ICPC-2 chapters $^{15}$ and CIRS domains $^{14}$ (table 1 ). There were some chronic conditions (eg, multisite cancer) that involved multiple systems. As these would usually be counted multiple times in different CIRS domains, we created an additional domain called 'Whole system', resulting in 15 CIRS domains instead of the usual 14. The ICPC-2 male and female genital system chapters (chapters $\mathrm{Y}$ and $\mathrm{X}$ ) were combined as they referred to the same body system, resulting in 16 ICPC-2 chapters (rather than the usual 17). This sample was previously shown to be representative of the age-sex distribution of patients at all GP encounters claimed (as items of service) through Medicare in 2008-2009. ${ }^{18}$

Using this large prospective study, we examined the effect of three different dimensions of measuring multimorbidity while controlling for other confounding variables. This is achieved through the structure of the study, by only changing one of the three variables at a time.

\section{Dimension 1: Does the way disease entities are defined} affect multimorbidity prevalence estimates?

To test this dimension, we defined disease entity in four different ways. First, each recorded/ticked chronic condition was treated as a separate disease entity. For the other three methods, we considered a disease entity to be a chapter/domain that was affected by at least one chronic condition in each of the three classification systems. Comparing the resulting multimorbidity prevalence estimates, we were able to test two research questions. First, whether counting different body systems affected by chronic conditions produces prevalence estimates comparable to counting individual chronic conditions. Second, whether counting the number of different CIRS domains, ICPC-2 chapters or ICD-10 chapters affected produces comparable prevalence estimates.

\section{Dimension 2: Does the minimum number of disease entities required to define multimorbidity affect multimorbidity prevalence estimates?}

We compared prevalence of multimorbidity using 2+ through to $6+$ disease entities. We also compared the agespecific prevalence of multimorbidity when it was defined as $2+$ and $3+$ disease entities, to see whether we could reproduce the 'S'-shaped curve when using the $2+$ definition and test whether using $3+$ provided greater differentiation among older patients, as found by Fortin $e t a l .{ }^{11}$

\section{Dimension 3: Does the number of chronic conditions} included in the study affect multimorbidity estimates?

We reduced the number of chronic conditions used, in order to simulate studies that were based on fewer chronic conditions. We used the 11 minimum chronic conditions as suggested by Diederichs et al), ${ }^{12}$ the 12 most prevalent chronic conditions in our study (hypertension, hyperlipidaemia, ischaemic heart disease, type 2 diabetes, obesity, osteoarthritis, chronic back pain, asthma, depression, anxiety, gastro-oesophageal reflux disease and malignant neoplasms) as suggested by Fortin $e t a l^{11}$ and the 24 listed chronic conditions with a tick box. We then compared these results with those generated using all diagnosed chronic conditions.

BEACH substudies have a single stage cluster design, with each GP having 30 patients clustered around them. The cluster effect was accounted for using SAS V.9.3.

\section{RESULTS}

Completed research packs were returned by 290 GPs $(77.3 \%)$ sampling 8707 patients. In total, $66.5 \%$ of patients $(n=5777)$ had at least one chronic condition and $33.7 \%(\mathrm{n}=2930)$ had none. The intracluster correlation coefficient was 0.121 for patients with at least one chronic condition.

Table 1 shows the proportion of patients with at least one chronic condition in each chapter/domain. For ICPC-2 and ICD-10, the 11 most prevalent chapters were body specific, with the non-body system-specific chapters being relatively uncommon. Prevalence estimates of patients with at least one chronic condition within a body system-specific ICD-10 and ICPC-2 chapter were remarkably similar, the top six chapters being in the same order, with no significant differences in the 
Table 1 Proportion of patients in GP waiting rooms that have at least one condition in each CIRS domain, ICPC-2 chapter or ICD-10 chapter

\begin{tabular}{|c|c|c|c|c|c|c|c|c|}
\hline CIRS domain & $\mathbf{n}$ & $\begin{array}{l}\text { Proportion of patients } \\
\text { in waiting room (\%, } \\
95 \% \text { Cls) }\end{array}$ & ICPC-2 chapter & $\mathbf{n}$ & $\begin{array}{l}\text { Proportion of patients } \\
\text { in waiting room }(\% \text {, } \\
95 \% \mathrm{Cl})\end{array}$ & ICD-10 chapter & $\mathbf{n}$ & $\begin{array}{l}\text { Proportion of patients } \\
\text { in waiting room (\%, } \\
95 \% \mathrm{Cl})\end{array}$ \\
\hline Vascular & 2934 & 33.7 (31.7 to 35.7 ) & K (Circulatory) & 2762 & 31.7 (29.8 to 33.6$)$ & 9 (Circulatory) & 2748 & 31.6 (29.7 to 33.5$)$ \\
\hline Musculoskeletal ${ }^{\star}$ & 2479 & 28.5 (26.6 to 30.4 ) & $\mathrm{T}$ (Endocrine†) & 2694 & 30.9 (29.2 to 32.7$)$ & 4 (Endocrine $\ddagger)$ & 2688 & 30.9 (29.1 to 32.7$)$ \\
\hline Endocrine $^{*}$ & 1840 & 21.1 (19.7 to 22.5 ) & P (Psychological) & 1953 & 22.4 (20.8 to 24.0 ) & $\begin{array}{l}5 \text { (Mental and } \\
\text { behavioural disorders) }\end{array}$ & 1910 & 21.9 (20.4 to 23.5$)$ \\
\hline Respiratory & 1195 & 13.7 (12.7 to 14.8$)$ & D (Digestive) & 1387 & 15.9 (14.7 to 17.2$)$ & 11 (Digestive) & 1296 & 14.9 (13.7 to 16.1$)$ \\
\hline Neurological & 542 & $6.2(5.4$ to 7.0$)$ & U (Urology) & 312 & 3.6 (3.0 to 4.2$)$ & 14 (Genitourinary) & 389 & 4.5 (3.8 to 5.2 ) \\
\hline Ophthalmological ${ }^{\star}$ & 444 & 5.1 (4.4 to 5.8 ) & N (Neurological) & 311 & 3.6 (3.1 to 4.1$)$ & 6 (Nervous system) & 318 & 3.7 (3.1 to 4.2 ) \\
\hline $\begin{array}{l}\text { Lower } \\
\text { gastrointestinal }\end{array}$ & 377 & 4.3 (3.7 to 4.9 ) & F (Eye) & 303 & 3.5 (2.9 to 4.1$)$ & 7 (Eye and adnexa) & 292 & 3.4 (2.8 to 3.9 ) \\
\hline Genitourinary & 351 & 4.0 (3.4 to 4.6 ) & S (Skin) & 294 & 3.4 (2.8 to 3.9 ) & $\begin{array}{l}12 \text { (Skin and } \\
\text { subcutaneous tissue) }\end{array}$ & 179 & 2.1 (1.7 to 2.4 ) \\
\hline Renal & 232 & 2.7 (2.2 to 3.2 ) & $\begin{array}{l}\text { A (General and } \\
\text { unspecified) }\end{array}$ & 134 & 1.5 (1.2 to 1.8$)$ & 18 (Symptomsł) & 105 & $1.2(0.9$ to 1.5$)$ \\
\hline \multirow[t]{6}{*}{ Whole system } & 39 & 0.4 (0.3 to 0.6$)$ & W (Pregnancy†) & 8 & 0.1 (0.0 to 0.2$)$ & 3 (Blood $\ddagger)$ & 58 & 0.7 (0.5 to 0.9$)$ \\
\hline & & & Z (Social problems) & 2 & $0.0(0.0$ to 0.1$)$ & 19 (Injury, poisoning $\ddagger$ ) & 58 & 0.7 (0.5 to 0.8$)$ \\
\hline & & & & & & $\begin{array}{l}8 \text { (Ear and mastoid } \\
\text { process) }\end{array}$ & 47 & 0.5 (0.4 to 0.7$)$ \\
\hline & & & & & & 17 (Congenitalł) & 23 & $0.3(0.2$ to 0.4$)$ \\
\hline & & & & & & 15 (Pregnancy & 3 & $0.0(0.0$ to 0.1$)$ \\
\hline & & & & & & $\begin{array}{l}16 \text { (Conditions- } \\
\text { perinatal period } \ddagger \text { ) }\end{array}$ & 2 & $0.0(0.0$ to 0.1$)$ \\
\hline
\end{tabular}

${ }^{*}$ CIRS: musculoskeletal and tegumental; endocrine, metabolic, breast; ophthalmological and otorhinolaryngology.

IICPC-2: endocrine, nutritional and metabolic; blood, blood forming organs and immune mechanism; ear and hearing; pregnancy, childbearing, family planning.

fICD-10: endocrine, nutritional and metabolic; musculoskeletal and connective tissue; symptoms, signs and abnormal clinical and laboratory findings; certain infectious and parasitic diseases

factors influencing health status and contact with health services; blood, blood forming organs and certain disorders involving the immune mechanism; injury, poisoning and certain other

consequences of external causes; congenital malformations, deformations and chromosomal abnormalities; pregnancy, childbirth and the puerperium; certain conditions originating from the

perinatal period.

CIRS, Cumulative IIIness Rating Scale; ICD, International Classification of Disease; ICPC, International Classification of Primary Care; GP, general practitioner. 
prevalence estimates for these six chapters. There were larger differences between estimates using CIRS and those from ICPC-2 and ICD-10. The major differences were due to CIRS splitting cardiovascular into vascular and cardiac domains, classifying cerebrovascular disease as neurological and classifying hyperlipidaemia in the vascular domain. In all systems, the most frequent chapters/domains were those relating to the: cardiac/vascular/circulatory; endocrine; musculoskeletal; psychological; digestive and respiratory systems.

Figure 1 shows the prevalence of multimorbidity among patients in the sample (representing those in a GP's waiting room) using different definitions of multimorbidity. The estimated prevalence of multimorbidity ranged from $47.4 \%$ when using 2+ individual chronic conditions to $2.8 \%$ when using 6+ ICPC-2 chapters. For all definitions using $3+$ disease entities or more, counting individual chronic conditions resulted in a significantly higher prevalence estimate than any of the grouped estimates. This difference increased proportionally as the minimum number of disease entities increased-the individual chronic conditions estimate was $23 \%$ higher than the ICPC-2 chapter estimate at $3+$ disease entities, through to $268 \%$ higher at $6+$ disease entities. Overall, there was no significant difference found between prevalence estimates using ICD-10, ICPC-2 and CIRS, from 2+ through to $6+$ disease entities.

Using the ICD-10 and ICPC-2 estimates, when multimorbidity was defined as two or more disease entities, about $44 \%$ of patients presenting to GPs were identified as multimorbid. This prevalence decreased with each increase in the number of disease entities required, with about $27 \%$ of patients being considered multimorbid for $3+$, about $15 \%$ for $4+, 7 \%$ for $5+$ and only $3 \%$ for $6+$ disease entities. There was nearly perfect concordance between patients identified as having multimorbidity using the ICD-10 and ICPC-2 classification systems. For example, when using the minimum of three disease entities as the definition of multimorbidity, over $99 \%$ of patients identified using ICD-10 were also identified using ICPC-2 and vice versa (table 2). There was also high concordance between ICPC-2/ICD-10 and CIRS. For every 12 patients identified as having multimorbidity with CIRS, 11 were also identified using ICPC-2/ICD-10 and vice versa.

Figure 2 shows multimorbidity prevalence estimates using the 2+ and the 3+ definitions across the different number of chronic conditions included. For all classification groups, the prevalence estimates derived when using Diederichs $e t$ als 11 chronic conditions were significantly lower than those using the 12 most prevalent chronic conditions, which in turn were significantly lower than the estimates based on all chronic conditions. Prevalence estimates based on the 12 most prevalent chronic conditions and on the 24 common chronic conditions (tick boxes) did not significantly differ, except that the 24 chronic conditions produced higher estimates when using $3+$ individual chronic conditions or 3+ CIRS domains.

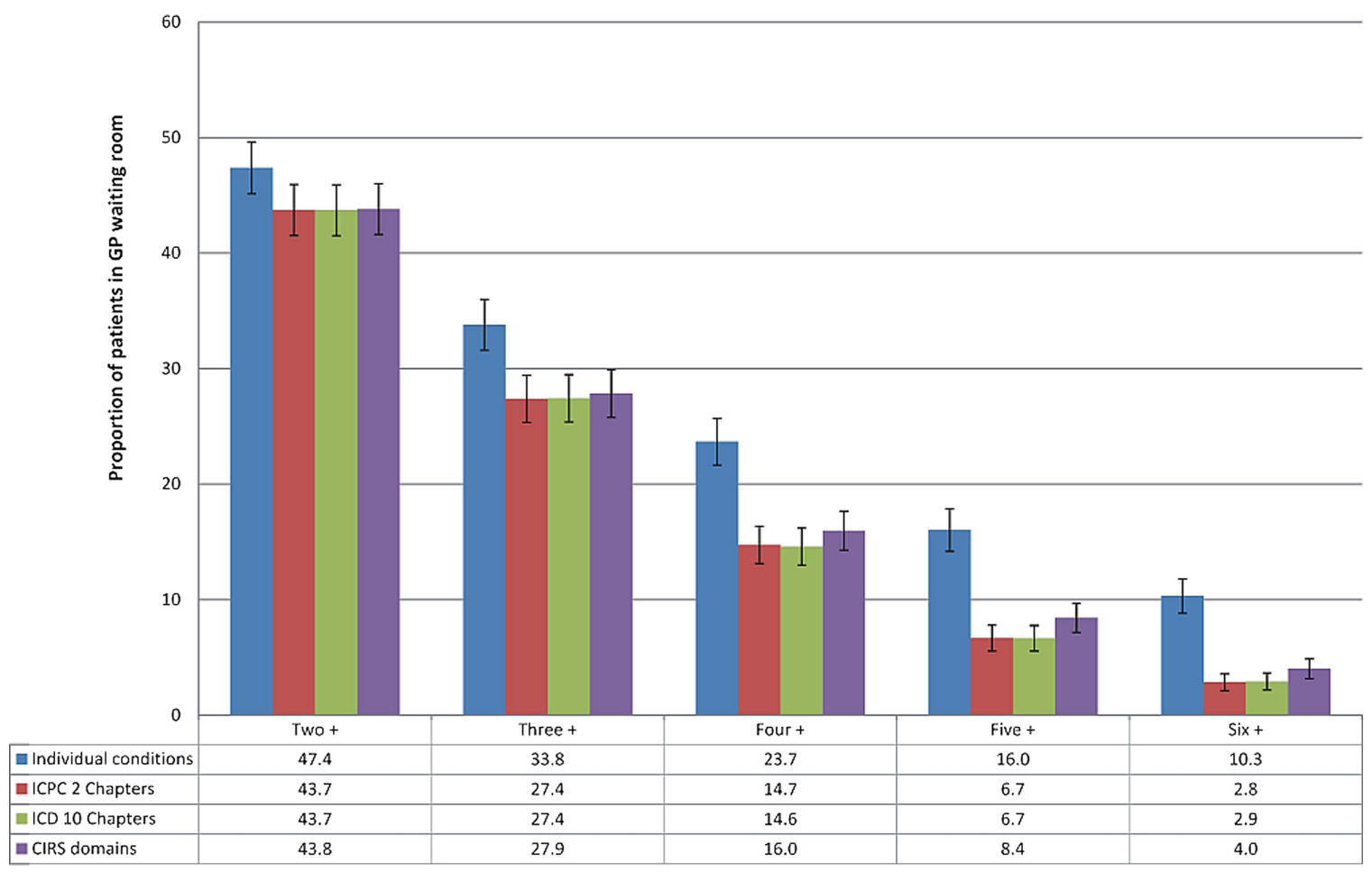

Figure 1 Multiple conditions within patients as defined by different classification systems (CIRS, Cumulative Illness Rating Scale; GP, general practitioners; ICD, International Classification of Disease; ICPC, International Classification of Primary Care). 
Table 2 Concordance of patients identified with multimorbidity (3+ definition) between ICPC-2, ICD-10 and CIRS

\begin{tabular}{|c|c|c|c|}
\hline & \multicolumn{3}{|c|}{$\begin{array}{l}\text { Proportion of patients identified as having multimorbidity using each classification system } \\
\text { (horizontal) that were also identified using other classification systems (vertical) (\%, } 95 \% \text { Cls) }\end{array}$} \\
\hline & ICPC-2 & ICD-10 & CIRS \\
\hline ICPC-2 & 100.0 & 99.1 (98.7 to 99.5$)$ & 92.1 (90.9 to 93.3$)$ \\
\hline ICD-10 & 99.3 (98.9 to 99.6$)$ & 100.0 & 91.9 (90.7 to 93.1$)$ \\
\hline CIRS & 93.7 (92.6 to 94.7$)$ & 93.3 (92.2 to 94.4$)$ & 100.0 \\
\hline
\end{tabular}

When using a restricted number of chronic conditions (ie, Diederichs et als list or Fortin et als 12 ) rather than all chronic conditions, the proportion of patients identified as having multimorbidity was significantly less when multimorbidity was defined as $3+$ than when defined as $2+$. For example, applying the $2+$ definition to ICPC-2 chapters, using the 12 most prevalent chronic conditions identified $79.4 \%$ of those identified as multimorbid using all chronic conditions. Using the 3+ ICPC-2 chapters definition, the 12 most prevalent conditions only identified $67.5 \%$. Similarly, using Diederichs et al's list with the $2+$ definition identified $54.5 \%$ and the $3+$ definition identified only $32.8 \%$ of those identified using all chronic conditions.

Figure 3 shows the age-specific multimorbidity prevalence estimates using the $2+$ and $3+$ definitions by individual chronic conditions and ICPC-2 chapters. Only the ICPC-2 chapters are presented as we have demonstrated that there was no significant difference between estimates derived using ICPC-2 chapters, ICD-10 chapters or CIRS domains. The age-specific prevalence using $2+$ individual chronic conditions and 2+ ICPC-2 chapters increased rapidly up to the 70-79 years age group, and remained steady in the older age groups. Compared with $2+$, the increase in prevalence started later for $3+$ individual chronic conditions (between 20-29 and 30-39 years of age). For 3+ ICPC-2 chapters, this increase started even later (between 30-39 and 40-49 years of age). For both the $3+$ measures, the prevalence did not plateau until 80 89 years of age, 10 years later than when using the 2+ definition.

\section{DISCUSSION}

This study has shown that multimorbidity prevalence estimates are independently affected by the number of chronic conditions collected in a study, how a disease entity is defined, and the minimum number of disease entities used to define multimorbidity. It has also demonstrated that health data classified to ICPC-2 chapters, ICD-10 chapters or CIRS domains produce similar multimorbidity prevalence estimates.

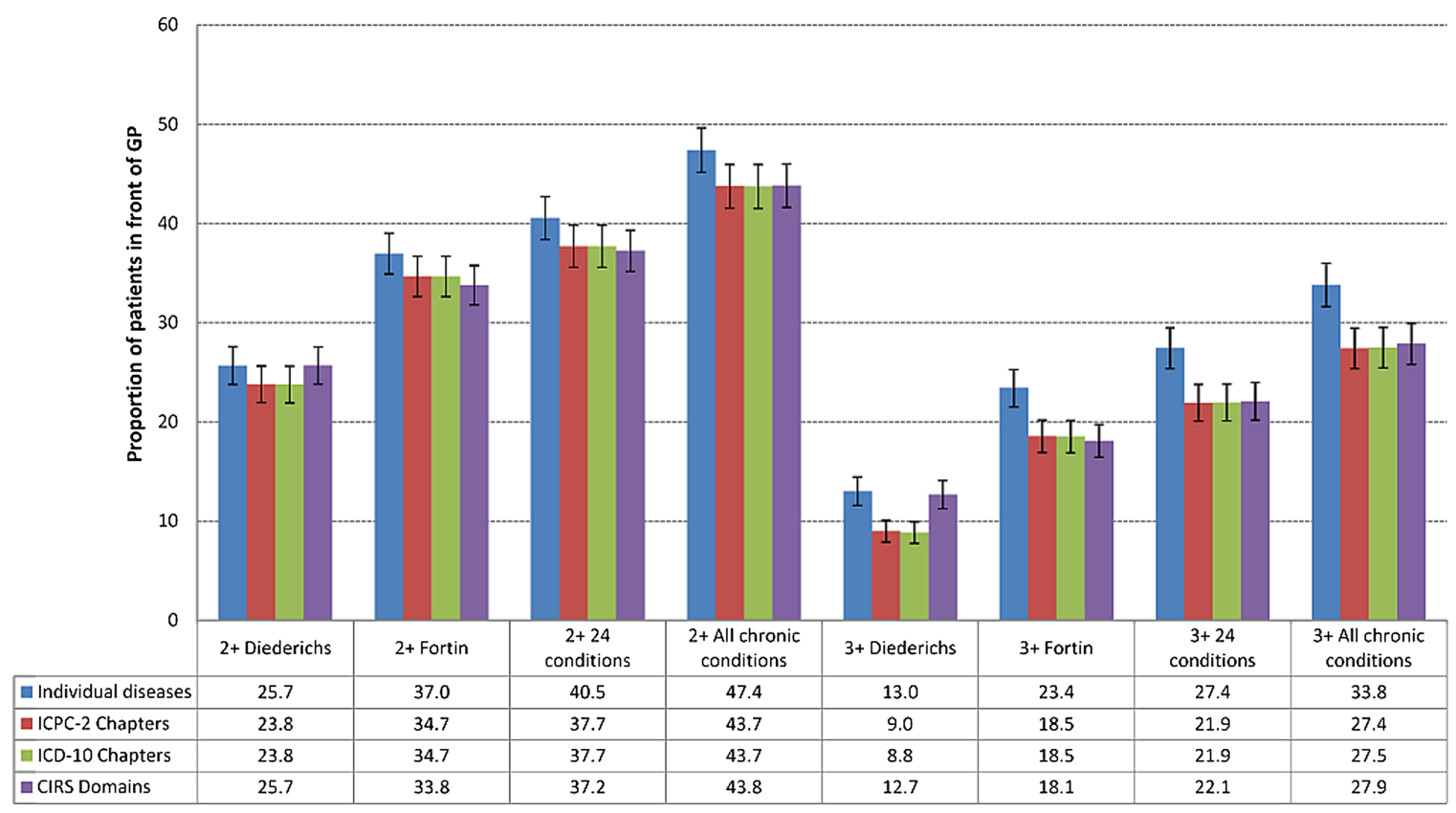

Figure 2 Estimated prevalence of multimorbidity by different classification systems and by whether $2+$ or $3+$ minimum number of disease entities was used (CIRS, Cumulative IIIness Rating Scale; GP, general practitioners; ICD, International Classification of Disease; ICPC, International Classification of Primary Care). 


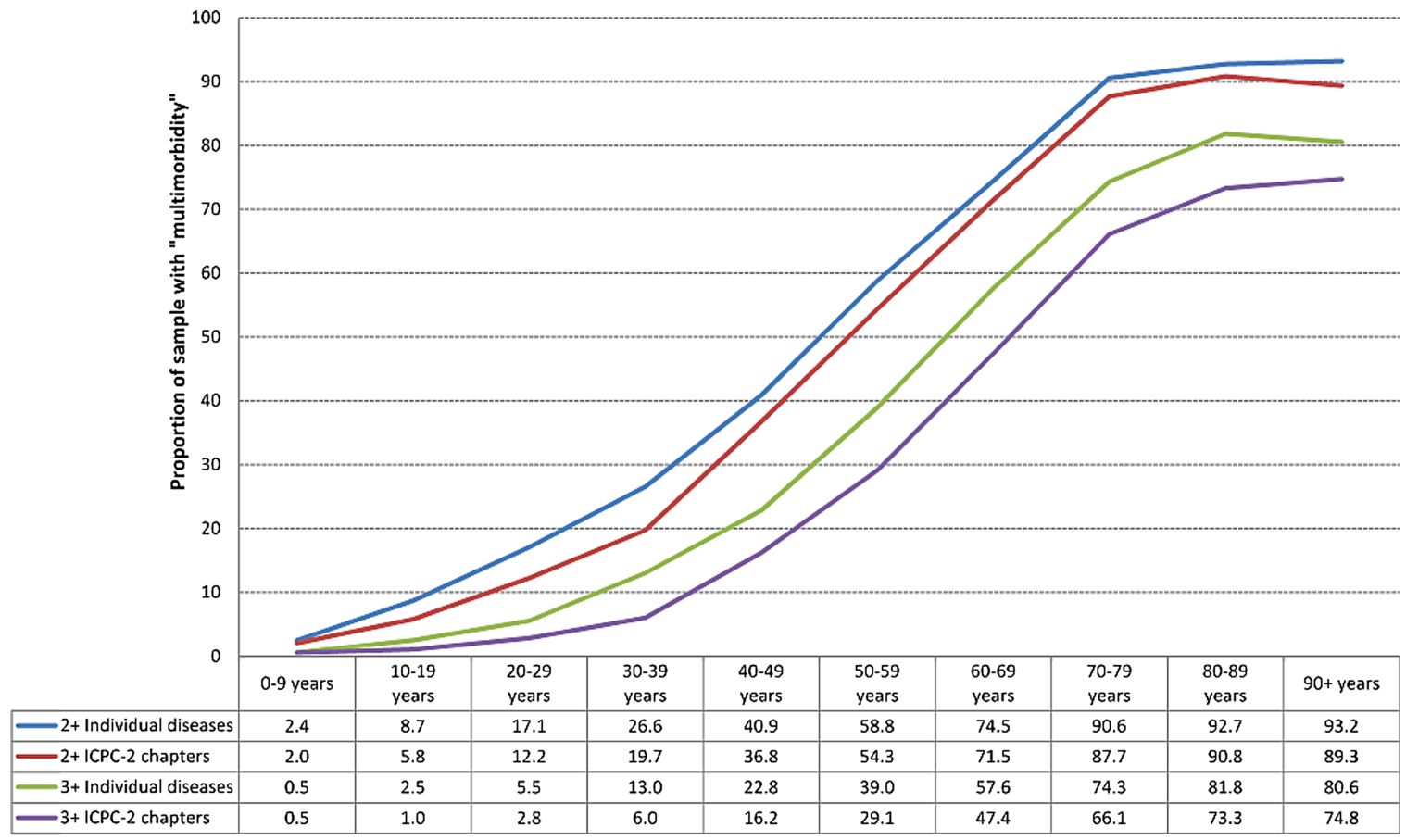

Figure 3 Patient age-specific prevalence of 'multimorbidity' (ICPC, International Classification of Primary Care).

Dimension 1: Does the way disease entities are defined affect multimorbidity prevalence estimates?

We found that when multimorbidity is defined as $2+$ disease entities, prevalence estimates are similar no matter how a disease entity is defined, be it an individual chronic condition or an ICPC-2 chapter, ICD-10 chapter or CIRS domain involving one or more chronic conditions. This means that studies that define multimorbidity as $2+$ can be compared even if the morbidity is classified differently. However, when multimorbidity is defined as $3+$ disease entities, using individual chronic conditions produces higher prevalence estimates than counting the different domains/chapters affected. We conclude that researchers should not compare results from studies using the 3+ definition when one study has used grouped chronic conditions (classified) and the other individual chronic conditions.

Our finding that chronic conditions were predominantly classified to body system-specific chapters/domains for all three classifications suggests that chapters/ domains could be used to represent the body systems affected. We also found no difference between the prevalence estimates produced with any of the three classification systems. Together, these results suggest that researchers may compare prevalence estimates from studies that count different ICPC-2 chapters, ICD-10 chapters or CIRS domains affected by chronic conditions. This allows researchers to draw data from primary care or hospital health records regardless of the classification system used (ICPC-2 or ICD-10) and know that results will be comparable to published studies that have used CIRS. $^{4} 13$
Dimension 2: Does the minimum number of disease entities required to define multimorbidity affect multimorbidity prevalence estimates?

We found that the higher the minimum number of different disease entities used to define multimorbidity, the lower the prevalence estimate. If multimorbidity is defined as 2+ disease entities, nearly every second person sitting in front of the GP would have multimorbidity, whereas using $3+$ decreased the estimate to nearly one in four. Like Fortin et al, we found that the 3+ definition provided greater differentiation in the older age groups than the 2+ definition. These results support their argument that using 2+ disease entities identifies such a large proportion of patients as having multimorbidity that it lacks the specificity to be useful, with a minimum of three disease entities arguably a better measure of multimorbidity.

\section{Dimension 3: Does the number of chronic conditions} included in the study affect multimorbidity estimates? As previous research suggests, ${ }^{11}{ }^{12}$ the number of chronic conditions studied affects the multimorbidity prevalence estimates-estimates based on a low number of chronic conditions being a fraction of those based on all chronic conditions. In our study, Diederichs et als list identified only half the patients identified with multimorbidity using all chronic conditions when using 2+, and only a third using 3+. Including the 12 most prevalent chronic conditions (suggested by Fortin $e t$ al), four of five multimorbid patients were identified using 2+ and two-thirds using $3+$. While both used a similar number of chronic conditions, Diederichs et $a l$ s list 
included the most prevalent chronic conditions in patients aged 65 years and over, whereas Fortin et al suggested the most prevalent overall conditions.

It is clear from these results that no matter how multimorbidity is defined, the list of chronic conditions suggested by Diederichs $e t a l$ as a minimum is not sufficient to reliably measure multimorbidity prevalence. Using the 12 most prevalent chronic conditions, as suggested by Fortin et al, does provide prevalence estimates that are reasonably close to those gained with all chronic conditions when using the $2+$ definition. However, when multimorbidity is defined as $3+$, the 12 most prevalent chronic conditions are not sufficient to measure multimorbidity. For the 3+ definition, ideally researchers should include all chronic conditions in their study.

This study has some limitations. We only included chronic conditions, whereas some authors have recently included acute conditions in their definition of multimorbidity. ${ }^{9}{ }^{21}$ Including acute conditions is understandable in a clinical setting, as they will temporarily increase the patient's complexity of care. However, where the goal is to measure the prevalence of multimorbidity to inform planning to meet the health resource requirements of these high-need patients, the use of only chronic conditions is logical.

Fortin et al suggest that when studying multimorbidity, one should also include a measure of severity. This study did not attempt to measure severity because of the limited space on the questionnaire and concerns that the additional burden on the GPs may reduce the response rate.

While our study was representative of patients at GP encounters, it should be remembered that patients are not representative of the population. Patients at GP encounters are generally older and therefore more likely to have a chronic condition. ${ }^{18}$

While our study was cross-sectional, the variables tested are relevant to all types of multimorbidity studies, be they cross-sectional, longitudinal, interview-based or based on a health record review.

Throughout this study, we have found that multimorbidity behaves quite differently when defined as $2+$ or $3+$ disease entities. With the 2+ definition, reasonable prevalence estimates could be obtained using only a dozen prevalent chronic conditions, regardless of how a disease entity was defined. With the $3+$ definition, the way the disease entity was defined was importantcounting individual chronic conditions produced significantly higher estimates than counting chapters/ domains. The number of chronic conditions studied was also important as studying a restricted number of chronic conditions produced significantly lower estimates than studying all chronic conditions. However, the prevalence estimates gained using 2+ were so encompassing that they lacked specificity-especially in older patients-whereas $3+$ provided greater specificity and more differentiation among the elderly patients.
These results suggest that the concepts of 2+ and 3+ multimorbidity are quite different. Rather than having both these concepts included under the same label, we propose adding the word 'complex' to those patients with 3+ chronic conditions from different body systems to clarify the meaning. 'Multimorbidity' would be defined as the "co-occurrence of two or more chronic conditions within one person without defining an index chronic condition." 'Complex multimorbidity' would be defined as the "co-occurrence of three or more chronic conditions affecting three or more different body systems within one person without defining an index chronic condition." In this way, we still have the more encompassing 2+ definition to compare with a previous work, while also being able to identify patients requiring additional care.

For consistency, we also propose a similar concept for comorbidity. We suggest that 'complex comorbidity' be defined as "the existence of two or more additional chronic conditions from two or more body systems different to that of the index chronic condition under study." This would mean that all patients with complex multimorbidity would also have complex comorbidity, the only difference being whether there is a chronic condition of interest.

There are advantages to using body systems affected (as represented by chapters/domains to which a chronic condition had been classified) rather than individual chronic conditions as 'disease entities'. Take, for example, two patients with three chronic conditions: patient A has peripheral vascular disease, hypertension and type 2 diabetes; patient B has depression, osteoarthritis and type 2 diabetes. The chronic conditions in patient A only affect two body systems while those in patient $\mathrm{B}$ affect three. According to our definitions, both would have multimorbidity, but patient B would also have complex multimorbidity. Patients identified with chronic conditions in 3+ body systems (complex multimorbidity) may be those whose care is more complex, as chronic conditions in different body systems are likely to compete for treatment, while the treatments of chronic conditions within the same system are more likely to be complementary. This is a similar concept to Piette and Kerr's ${ }^{22}$ idea of concordant and discordant comorbidity.

Counting the body systems affected also provides an estimate of the specialist types that may be involved in the care of the patient. This is important for healthcare planning as it reduces double counting of chronic conditions that may be referred to the same specialist type; for example, a patient with depression and anxiety may be referred to one psychiatrist (not two). It also identifies patients who may need assistance with coordination of specialist care, as the healthcare of patients with multimorbidity is more likely to be poorly coordinated. ${ }^{2324}$ 


\section{CONCLUSION}

For the first time, a single large prospective study has been used to test the effect of the way multimorbidity is measured on prevalence estimates, while controlling for other variables, using the same data for all measures. This is not possible with systematic reviews. We have shown that multimorbidity behaves differently when defined as 2+ disease entities, as compared with when it is defined as $3+$ disease entities. To address this, we recommend that

- 'Multimorbidity' be defined as the "co-occurrence of two or more chronic conditions within one person without defining an index chronic condition";

- 'Complex multimorbidity' be defined as the "co-occurrence of three or more chronic conditions affecting three or more different body systems within one person without defining an index chronic condition."

This study provides some evidence that complex multimorbidity is a more useful measure of multimorbidity as it results in a lower prevalence estimate and shows greater differentiation among older patients. However, further research is needed to assess whether 'complex multimorbidity' is indeed better than alternative measures of multimorbidity (such as counting individual chronic conditions, measures of severity, etc) in identifying patients with greater healthcare resource use, complexity of care, lower quality of life and overall severity of illness.

Acknowledgements We wish to thank the GPs who participated, and the Australian Government Department of Health and Ageing for the random sample of GPs and data pertaining to the sample frame from which the samples were drawn.

Contributors All authors contributed to the conception and design of the substudy, collection of the data, interpretation of the data and critical revision of important intellectual content. $\mathrm{CH}$ conceptualised the study, supervised the data entry, performed the statistical analysis and wrote the first draft of the article and all redrafts. HB, GM and JH were all involved in the revision of all drafts of the paper. All authors approved the final version. $\mathrm{CH}$ is the study guarantor.

Funding Between 2008 and 2010 the BEACH programme, of which this substudy is a part, was funded by the Australian Government Department of Health and Ageing, the Australian Government Department of Veterans' Affairs, Australian Institute of Health and Welfare, National Prescribing Service, AstraZeneca Pty Ltd (Australia), Janssen-Cilag Pty Ltd, Merck, Sharpe and Dohme (Australia) Pty Ltd, Pfizer Australia Pty Ltd, Abbott Australasia Pty Ltd, Sanofi-Aventis Australia Pty Ltd, and Novartis Pharmaceuticals Australia Pty Ltd. The SAND substudy reported in this paper was commissioned by the Family Medicine Research Centre.

Ethics approval The BEACH programme and this sub-study were approved by the Human Research Ethics Committee of the University of Sydney (Reference No. 11428) and the Ethics Committee of the Australian Institute of Health and Welfare.

Provenance and peer review Not commissioned; externally peer reviewed. Data sharing statement No additional data are available.
Open Access This is an Open Access article distributed in accordance with the Creative Commons Attribution Non Commercial (CC BY-NC 3.0) license, which permits others to distribute, remix, adapt, build upon this work noncommercially, and license their derivative works on different terms, provided the original work is properly cited and the use is non-commercial. See: http:// creativecommons.org/licenses/by-nc/3.0/

\section{REFERENCES}

1. Feinstein AR. Clinical judgment. Baltimore, MD: Williams and Wilkins, 1967.

2. van den AM, Buntinx F, Roos S, et al. Problems in determining occurrence rates of multimorbidity. J Clin Epidemiol 2001;54:675-9.

3. United Nations. World population ageing: 1950-2050. UNITED NATIONS PUBLICATIONS, 2001. [cited 28 Oct 2013]. http://www. un.org/esa/population/publications/worldageing19502050/

4. Britt HC, Harrison CM, Miller GC, et al. Prevalence and patterns of multimorbidity in Australia. Med J Aust 2008;189:72-7.

5. Fortin M, Soubhi H, Hudon $\mathrm{C}$, et al. Multimorbidity's many challenges. BMJ 2007;334:1016-17.

6. Starfield B. Challenges to primary care from co- and multi-morbidity. Prim Health Care Res Dev 2011;12:1-2.

7. Schellevis FG, van d V, van de LE, et al. Comorbidity of chronic diseases in general practice. J Clin Epidemiol 1993;46:469-73.

8. Fortin M, Bravo G, Hudon C, et al. Prevalence of multimorbidity among adults seen in family practice. Ann Fam Med 2005;3:223-8.

9. Le Reste JY, Nabbe P, Manceau B, et al. The European General Practice Research Network presents a comprehensive definition of multimorbidity in family medicine and long term care, following a systematic review of relevant literature. J Am Med Dir Assoc 2013;14:319-25.

10. Stewart M, Fortin M, Britt HC, et al. Comparisons of multi-morbidity in family practice-issues and biases. Fam Pract 2013;30:473-80.

11. Fortin M, Stewart M, Poitras ME, et al. A systematic review of prevalence studies on multimorbidity: toward a more uniform methodology. Ann Fam Med 2012;10:142-51.

12. Diederichs C, Berger K, Bartels DB. The measurement of multiple chronic diseases - a systematic review on existing multimorbidity indices. J Gerontol A Biol Sci Med Sci 2011;66:301-11.

13. Brett T, Arnold-Reed D, Popescu A, et al. Multimorbidity in patients attending two Australian Primary Care Practices. Ann Fam Med 2013;11:535-42.

14. Hudon $\mathrm{C}$, Fortin $\mathrm{M}$, Soubhi $\mathrm{H}$. Abbreviated guidelines for scoring the Cumulative IIIness Rating Scale (CIRS) in family practice. J Clin Epidemiol 2007;60:212.

15. Classification Committee of the World Organization of Family Doctors. ICPC-2: International Classification of Primary Care. 2nd edn. Oxford: Oxford University Press, 1998.

16. World Health Organisation. International Statistical Classification of Diseases and Related Health Problems 10th Revision. 2013. [cited 28 Oct 2013]. http://apps.who.int/classifications/icd10/browse/2010/en

17. Britt H, Miller GC, Henderson J, et al. General practice activity in Australia 2012-13. Sydney: Sydney University Press, 2013.

18. Harrison $\mathrm{C}$, Britt $\mathrm{H}$, Miller $\mathrm{G}$, et al. Prevalence of chronic conditions in Australia. PLOS ONE 2013;8:e67494.

19. O'Halloran J, Miller GC, Britt H. Defining chronic conditions for primary care with ICPC-2. Fam Pract 2004;21:381-6.

20. Britt $\mathrm{H}$, Miller G. ICPC PLUS: an extended version of the International Classification of Primary Care for computerised clinical systems. Cambridge, Worcester: Primary Care Specialist Group of the British Computer Society, 1996.

21. Bayliss EA, Edwards AE, Steiner JF, et al. Processes of care desired by elderly patients with multimorbidities. Fam Pract 2008;25:287-93.

22. Piette JD, Kerr EA. The impact of comorbid chronic conditions on diabetes care. Diabetes Care 2006;29:725-31.

23. Wolff JL, Starfield B, Anderson G. Prevalence, expenditures, and complications of multiple chronic conditions in the elderly. Arch Intern Med 2002;162:2269-76.

24. Schoen C, Osborn R, Squires D, et al. New 2011 survey of patients with complex care needs in eleven countries finds that care is often poorly coordinated. Health Aff (Millwood) 2011;30:2437-48 